\title{
Frequency Up-Conversion of an Intense Laser Beam Producing Gas Ionization in a Cavity
}

\author{
K. Hizanidis, J.L. Vomvoridis, J.T. Mendonca* and D.J. Frantzeskakis** \\ Department of Electrical and Computer Engineering, National Technical University \\ of Athens, 15773 Athens, Greece \\ * Department of Physics, Instituto Superior Tecnico, 1096 Lisboa Codex, Portugal \\ ** Department of Physics, University of Athens, 15784 Athens, Greece
}

\begin{abstract}
Frequency up-conversion of a laser beam causing gas ionization in a cavity is considered. A simple ionization model, based on one-photon and multi-photon processes is used. The WKB method is utilized to provide the slow evolution of the field amplitude. The analytical results thus obtained are presented and contrasted with the exact numerical results where all the time scales are present. The evolution of the wave frequency and its upshift predicted by the WKB method are in very good agreement with the numerical results. The same applies, though to a lesser degree, for the field energy loss as well.
\end{abstract}

\section{INTRODUCTION}

Photon acceleration [1], i.e., the adiabatic frequency up-shift of wave-packets propagating in nonstationary media, has recently become the subject of an increasing interest [2-4], due to its implication in the search for new plasma-based radiation sources [5]. The so-called flash ionization effect, i.e., the frequency up-conversion of electromagnetic radiation propagating through a gas which is converted to a plasma via ionization, is one of the mechanisms of photon acceleration [6]. This effect, which was first demonstrated experimentally by Yablonowitch [7-8] with use of a laser beam, has been observed recently in the microwave region as well [9-11]. Theoretical studies concerning the wave propagation in a switched plasma medium, have also been performed [12-17].

In the usual modelling of the flash ionization effect, an electromagnetic wave propagating in a neutral gas, exhibiting a relative dielectric constant nearly equal to 1 , is considered. During the transition from the gas to the plasma state, the aforementioned wave experiences a reduction of the relative dielectric constant to the value $1-\omega_{p}^{2} / \omega^{2}$, where $\omega$ is the field frequency and $\omega_{p}$ is the time-varying plasma frequency. In this way, a significant change of the initial frequency $\omega_{0}$ of the wave occurs, since, theoretically, the new frequency is estimated to be $\omega=\sqrt{\omega_{0}^{2}+\omega_{p}^{2}}$. When the ionization takes place in a microwave cavity filled with a gas, the frequency $\omega_{0}$ corresponds to the initial value of the frequency of the field contained in the cavity. In this case, as it is demonstrated experimentally $[7-8,10]$, the frequency up-shift is always lower than the theoretically estimated one. A consistent explanation of the aforementioned discrepancy has been presented recently [17].

In the present work, the frequency up-conversion of a laser beam causing gas ionization in a cavity is considered. A simple ionization model, based on one-photon and multi-photon processes is used. The WKB method is utilized to provide the slow evolution of the field amplitude. This method exhibits the advantage of leading to analytical results which can easily provide information about the main dependencies of the evolution of the frequency upshift and the produced free electron density on the basic parameters of the system, that is, initial field amplitude, gas density and ionization time of the multi-photon ionization process chosen.

The paper is arranged as follows: In Sec. II, the one-dimensional wave equation for the electric field intensity, together with the model for the ionization growth rate, are presented. The solutions of these coupled differential equations by means of the lowest order WKB 
approximation for the one- and two-photon ionization processes are derived in Sec. III. Finally, in Sec. IV, the approximate WKB results are compared with the exact numerically obtained ones.

\section{PHYSICAL MODEL}

We consider a microwave cavity containing a gas at constant pressure and temperature. A moderately intense monochromatic electromagnetic wave, produced by a laser source, ionizes the gas at a particular ionization rate set by the physical conditions of the gas itself and the intensity of the laser light. For the sake of simplicity of the model in hand, we fix the ionization rate $T_{i o n}$ as compared to the injected laser frequency, $\omega_{0}=k c$, at a constant value (representative of the experiments and the simulations cited in the literature) throughout the examples to be presented. In the one-dimensional case of a transverse linearly polarized wave, the electric field intensity is of the form $\mathbf{E}=\mathbf{e}_{\mathbf{y}} E(x, t)$ and the wave equation is $c^{2} \partial^{2} E / \partial t^{2}-\partial^{2} E / \partial x^{2}=\epsilon_{0}^{-1} \partial J / \partial t$, where $c$ and $\epsilon_{0}$ are the velocity of light and the dielectric constant in vacuum respectively. As far as the driving term in the wave equation is concerned, the current density can be expressed as $J=-e n\langle v\rangle$, in terms of the density $n$ and the average velocity $\langle\nu\rangle$ of the plasma electrons ( $e$ is the electron charge). In the non-relativistic limit, where velocity $v$ and momentum $p=m v$ ( $m$ is the electron mass) are practically identical quantities, the current density is proportional to the momentum density, as $J=-(e / m) n\langle p\rangle$, hence its time derivative can be immediately related to the force density. Under these assumptions, the wave equation is reduced to the form [17],

$$
\left(c^{2} \frac{\partial^{2}}{\partial t^{2}}-\frac{\partial^{2}}{\partial x^{2}}\right) E(x, t)=\omega_{p}^{2}(t) E(x, t)
$$

where $\omega_{p}^{2}=e^{2} n / \in_{0} m$ is the time-varying plasma frequency.

In order to investigate the nonlinear dynamics of the interaction, a model for the electron density growth rate $\partial n / \partial t$ has to be introduced. In general, this growth rate is a complicated function of the field $E(x, t)$ determined by the combination of the processes of atomic-electronphoton kinetics. In the present work the following simple model $\partial n / \partial t \propto\left(n_{\infty}-n\right)|A|^{2 N}$ is used, where $A$ is component of the vector potential parallel to the transverse electric field in hand $(E=$ $\partial \mathrm{A} / \partial \mathrm{t}), n_{\infty}$ is the time-asymptotic value of the plasma density (that is, the density of the gas, if the latter is assumed to be fully ionized) and $N=I, 2, \ldots$ corresponds to one-, two-photon, etc, ionization processes. In terms of the instantaneous plasma frequency, the ionization model used is as follows,

$$
\frac{\partial \omega_{p}^{2}}{\partial t}=\left.\sigma_{N}\left(\omega_{p \infty}^{2}-\omega_{p}^{2}\right)\right|_{A} ^{2 N}
$$

where $\sigma_{N}$ is the parameter which is related to the $\mathrm{N}$-photon ionization rate (or cross section) and it will be considered as constant in the range of values the laser light frequency and intensity varies (upshifts) during the ionization process. It should be emphasized that the one-photon process may not coincide in frequency space with other multi-photon processes and, furthermore, it might be the prevailing ionization mechanism irrespective of frequency in the same gas.

The laser frequency is considered to be in the range $10^{14}-10^{15} \mathrm{~Hz}$ and the ionizable gas is such as nitrogen, neon or helium at a density in the range $10^{25}-10^{26} \mathrm{~m}^{-3}$. Since the ionization potentials for these gases are few volts, or tens of volts, the electrons are born omni-directionally with velocities (randomly oriented) moving at in the range of $10^{-6} \mathrm{~ms}^{-1}$. During the ionization process modelled by (1) with ionization times in the range $T_{i o n}=1 p s-1 n s$, the electrons can move in a distance $(1 \mu \mathrm{m}-1 \mathrm{~mm})$ much larger than the respective wavelegth of the laser light. Therefore, for a cavity (gas cell) with dimensions in that range or larger, spatial variations in the formed plasma density are insignificant. On the other hand, for laser light with wavelegth much smaller than the dimensions of the gas cell and the respective spatial pulse-width, spatial variations in the field amplitude are being averaged out during the ionization process. Thus, (1) can be rewritten in terms of the spatially independent envelope functions $F(t)$ and $G(t)$ for the electric field and the 
vector potential respectively and the quantity $|A|$ in the RHS of (2) is interpreted as the spatially averaged vector potential (the coefficients $1 / 2,3 / 8$, etc, this averaging yields, are suppressed by redefining the ionization parameter $\sigma_{i t}$ ). Therefore, the partial temporal derivatives are now replaced by the ordinary ones, the spatial second derivative in (1) is replaced by $-k^{2}$ and the electric field and the vector potential by their respective envelope functions. Thus, (1) is reduced to the following ordinary differential equation,

$$
\frac{d^{2} F}{d t^{2}}+k^{2} c^{2} F+\omega_{p}^{2}(t) F=0
$$

Upon introducing now the following normalized quantities, $\tau=k c t, \quad \varepsilon=e F / m k^{2} c$, $\alpha=e .4 / m, \rho=\omega_{p}^{2} / \omega_{p \infty}^{2}$, equations (3) and (2) can respectively be put in the following form:

$$
\begin{aligned}
& \frac{d^{2} \varepsilon}{d t^{2}}+\left(1+\kappa_{o}^{2} \rho\right) \varepsilon=0 \\
& \frac{d \rho}{d \tau}=\delta_{0}(1-\rho) \alpha^{2 N}
\end{aligned}
$$

Notice that in (4) and (5) the following equation holds, $\varepsilon=-d \alpha / d \tau$, while the constants $\kappa_{o}^{2}, \delta_{0}$ are given by $\kappa_{0}^{2}=\omega_{p \infty}^{2} / k^{2} c^{2}, \delta_{0}=1 /\left(T_{i c h}^{(N)} k c\right)$, where $T_{i o n}^{(N)}=\sigma_{N}^{-1}(e / m c)^{2 N}$ is the ionization time for $N$-photon ionization process.

\section{WKB ANALYSIS}

Equations (4) and (5) can be solved approximately using the WKB method: Initially, it is assumed that the normalized vector potential is of the form $\alpha(\tau)=\hat{\alpha}(\tau) \exp (i \varphi(\tau))$, with the time derivative of the phase $\varphi(\tau)$ being the normalized instantaneous frequency $v(\tau)$, i.e., $d \varphi / d \tau=v$. Then, it is easily seen that, to the leading order, the system of (4) and (5) yield the following equation:

$$
\frac{d}{d \tau}\left[\hat{\alpha}^{2}(\tau) v^{3}(\tau)\right]=0
$$

This result shows that the quantity $\hat{\alpha}^{2}(\tau) v^{3}(\tau)$ is time-invariant and is equal to its initial (constant) value, i.e., $\hat{\alpha}^{2}(\tau) v^{\hat{3}}(\tau)=\alpha_{o i}^{2}$ (note that $v_{n}=1$ ). The existence of this invariant implies that

$$
v^{2}(\tau)=1+\kappa_{o}^{2} \rho(\tau)
$$

Use of $(7)$ leads to the determination of the normalized vector potential envelope $\hat{\alpha}(\tau)$ :

$$
\hat{\alpha}(\tau)=\alpha_{o i}\left[1+\kappa_{o}^{2} \rho(\tau)\right]^{-3 / 4}
$$

Upon combining eqs. (5) and (8), one can readily obtain a first-order differential equation for the normalized density $\rho(\tau)$ for the one-photon ionization process $(N=1)$ :

$$
\frac{d \rho}{d \tau}=\frac{1}{2} \delta_{o} \alpha_{o i}^{2} \frac{1-\rho(\tau)}{\left[1+\kappa_{o}^{2} \rho(\tau)\right]^{3 / 2}}
$$

Equation ( 9 ) can readily be integrated, leading to the following result:

$$
\begin{aligned}
\tau= & \left(\frac{1}{2} \delta_{0} \alpha_{0}^{2}\right)^{-2}\left\{\left(1+\kappa_{0}^{2}\right)^{3 / 2} \ln \frac{2+\kappa_{0}^{2}[1+\rho(\tau)]+2 \sqrt{1+\kappa_{0}^{2}} \sqrt{1+\kappa_{0}^{2} \rho(\tau)}}{[1-\rho(\tau)]\left[2+\kappa_{0}^{2}+2 \sqrt{1+\kappa_{0}^{2}}\right]}\right. \\
& \left.-2\left(1+\kappa_{0}^{2}\right) \sqrt{1+\kappa_{0}^{2} \rho(\tau)}-\frac{2}{3}\left(1+\kappa_{0}^{2}\right)^{3 / 2}+2 \kappa_{0}^{2}+\frac{8}{3}\right\}
\end{aligned}
$$


In the same way, for the two-photon ionization process $(N=2)$, the following equation for the density $\rho(\tau)$ can readily be obtained,

$$
\frac{d \rho}{d \tau}=\frac{3}{8} \delta_{o} \alpha_{o i}^{4} \frac{1-\rho(\tau)}{\left[1+\kappa_{o}^{2} \rho(\tau)\right]^{3}}
$$

which, by direct integration, leads to the following result:

$$
\tau=\left(\frac{1}{2} \delta_{o} \alpha_{o i}^{2}\right)^{-1}\left\{\left(1+\kappa_{o}^{2}\right)^{3}\left[\ln \frac{1}{1-\rho(\tau)}-\rho(\tau)\right]+\rho\left(\tau\left\{1-\frac{k_{o}^{4}}{6}\left(9+3 \kappa_{o}^{2}+2 \rho(\tau)\right)\right]\right\}\right.
$$

\section{RESULTS AND DISCUSSION}

The basic equations which govern the ionization process, namely (4) and (5) are integrated numerically and the results are contrasted with those obtained by directly estimating the slow variation of the basic parameters of the problem from (7), (9), (10) and (12). The initial conditions imposed are $\hat{\alpha}(\tau=0)=\alpha_{o i} \cos \left(\varphi_{0}\right), \varepsilon(\tau=0)=\alpha_{o i} \sin \left(\varphi_{0}\right), \rho(\tau=0)=0$, where $\varphi_{0}$ is the initial phase of the incident wave. It should be emphasized that the choice of the initial phase is of extremely minor importance as far as the results is concerned (that is, it represents a displacement of the time scale). The results are summarized in Figs. 1-4:
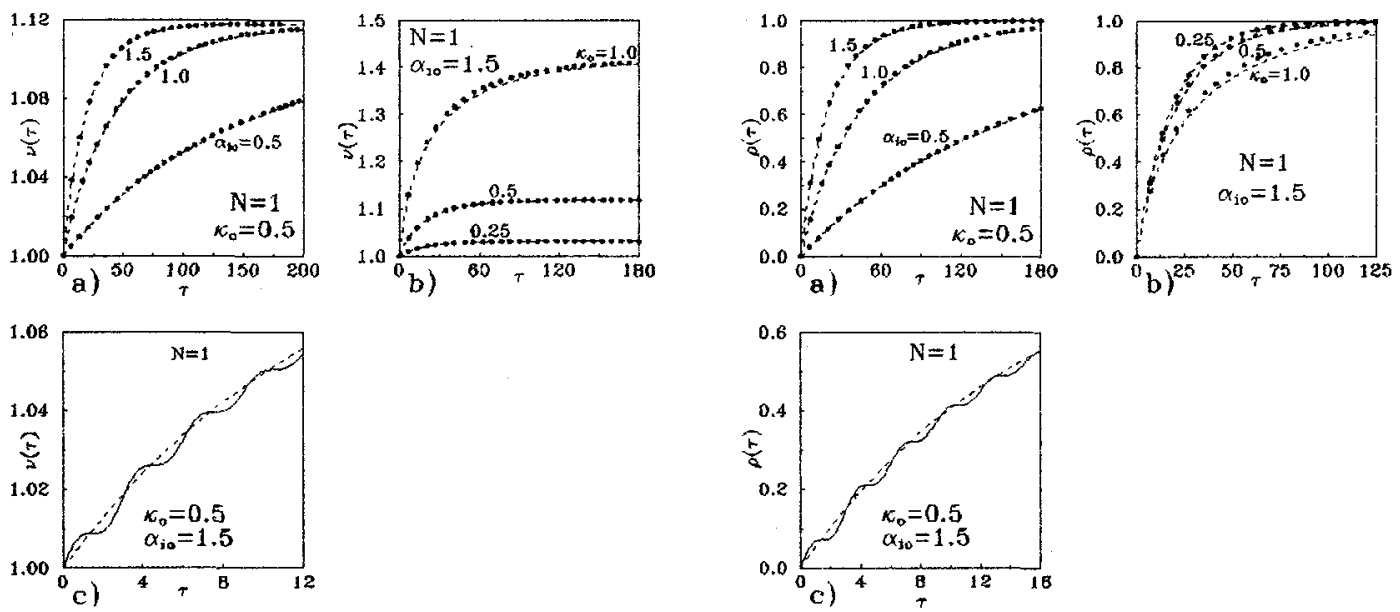

Figure 1: Frequency $v$ as a function of time $\tau$, for the one-photon ionization process: $(\alpha)$ For $\alpha_{i 0}=0.5,1.0$, 1.5 and $\kappa_{o}=0.5 ;(\mathrm{b})$ For $\kappa_{0}=0.25,0.5,1.0$ and $\alpha_{i o}=1.5$. The solid dots and the dashed curves refer to the WKB and the exact numerical solutions respectively. (c) Detail for a particular choice of $\alpha_{i o}$ and $\kappa_{0}$; the solid and the dashed curves correspond to the exact numerical and the WKB solutions respectively.

Figure 2: Plasma density $\rho$ as a function of time $\tau$ for the one-photon ionization process: ( $\alpha$ ) For $\alpha_{i o}=0.5,1.0$ and 1.5 and $\kappa_{o}=0.5$; (b) For $\kappa_{o}=0.25,0.5,1.0$ and $\alpha_{i o}=1.5$. The solid dots and the dashed curves refer to the WKB and the exact numerical solutions respectively. (c) Detail for a particular choice of $\alpha_{i o}$ and $\kappa_{o}$; the solid and the dashed curves correspond to the exact numerical and the WKB solutions.

The normalized frequency $v$, as a function of the normalized time $\tau$, for the one-photon $(N=1)$ ionization process, is shown in Fig. 1 . For a fixed value of the plasma parameter $\kappa_{o}\left(\kappa_{o}=\infty_{p}\right.$ $\alpha / k c=0.5$ ), the time-asymptotic value of $v$ is independent of the initial normalized field amplitude $\alpha_{i o}$, as shown in Fig. 1a for three different values of $\alpha_{i o}\left(\alpha_{i o}=0.5,1.0\right.$ and 1.5$)$, and takes the value 1.118. On the other hand, for three different values of the plasma parameter, $\kappa_{0}=0.25,0.5$ and 1.0 and fixed value of the initial normalized field amplitude $\alpha_{i o}\left(\alpha_{i o}=1.5\right)$, the time-asymptotic value of $v$ takes the values $1.03,1.12$ and 1.4 respectively (Fig. 1b). As it is readily observed, the smaller $\alpha_{i o}$ is, the bigger the saturation time is; this result is expected due to the fact that the ionization growth 
rate is proportional to a power of the field amplitude. The solid dots and the dashed curves in Figs. $1 \mathrm{a}$ and $\mathrm{lb}$ refer to the WKB and the exact numerical solutions respectively. As it is seen, the results are almost identical. The differences between the approximate analytical solution and the numerical solution is shown in Fig. $1 \mathrm{c}$ for a particular choice of $\alpha_{i o}$ and $\kappa_{0}$ : the solid and the dashed curves correspond to the numerical and the WKB solutions respectively.

The results obtained for the normalized plasma density $\rho$, are similar with the ones obtained for the normalized frequency $v$, as it is shown in Fig. 2: In Fig. 2a, the density $\rho$, as a function of the normalized time $\tau$, for the one-photon $(N=1)$ ionization process, is presented for three different values of the initial normalized field amplitude $\left(\alpha_{i o}=0.5,1.0\right.$ and 1.5$)$ and fixed value of $\kappa_{o}$ $\left(\kappa_{O}=0.5\right)$. The density dependence of the plasma parameter is shown in Fig. $2 \mathbf{b}$, for three values of $\kappa_{o}$, namely $0.25,0.5$ and 1.0 , and fixed value of the initial normalized field amplitude $\left(\alpha_{i o}=1.5\right)$. As previously, the solid dots and the dashed curves refer to the WKB and the exact numerical solutions respectively. A detail for a particular choice of $\alpha_{i o}$ and $\kappa_{o}$ is shown in Fig. 2c, where the solid and the dashed curves correspond to the exact numerical and the WKB solutions.

During the ionization process, there is an energy loss of the ionizing electromagnetic field, due to the electrons motion and the subsequent development of conductivity current. The net relative energy loss can be expressed as $1-\varepsilon^{2} / \varepsilon_{0}{ }^{2}$, wher $\varepsilon=\xi(\tau)$ refers to the amplitude of the slowly varying electric field envelope, i.e., $\varepsilon(\tau)=\left\{[d \alpha(\tau) / d t\}^{2}+v^{2}(\tau) \alpha^{2}(\tau)\right\}{ }^{1 / 2}$, while $\varepsilon_{0}=\varepsilon(\tau=0)$. The WKB result for the aforementioned net relative energy loss during $\alpha$ one-photon $(N=1)$ ionization process is shown in Fig. 3a, for three different values of the initial normalized field amplitude $\left(\alpha_{i o}=0.5,1.0\right.$ and 1.5 ) and fixed value of the plasma parameter $\left(\kappa_{0}=0.5\right)$. As it is seen, the time-asymptotic value of the net relative energy loss is near $10 \%$, and the smaller the initial field amplitude is, the longer it takes to be reached.
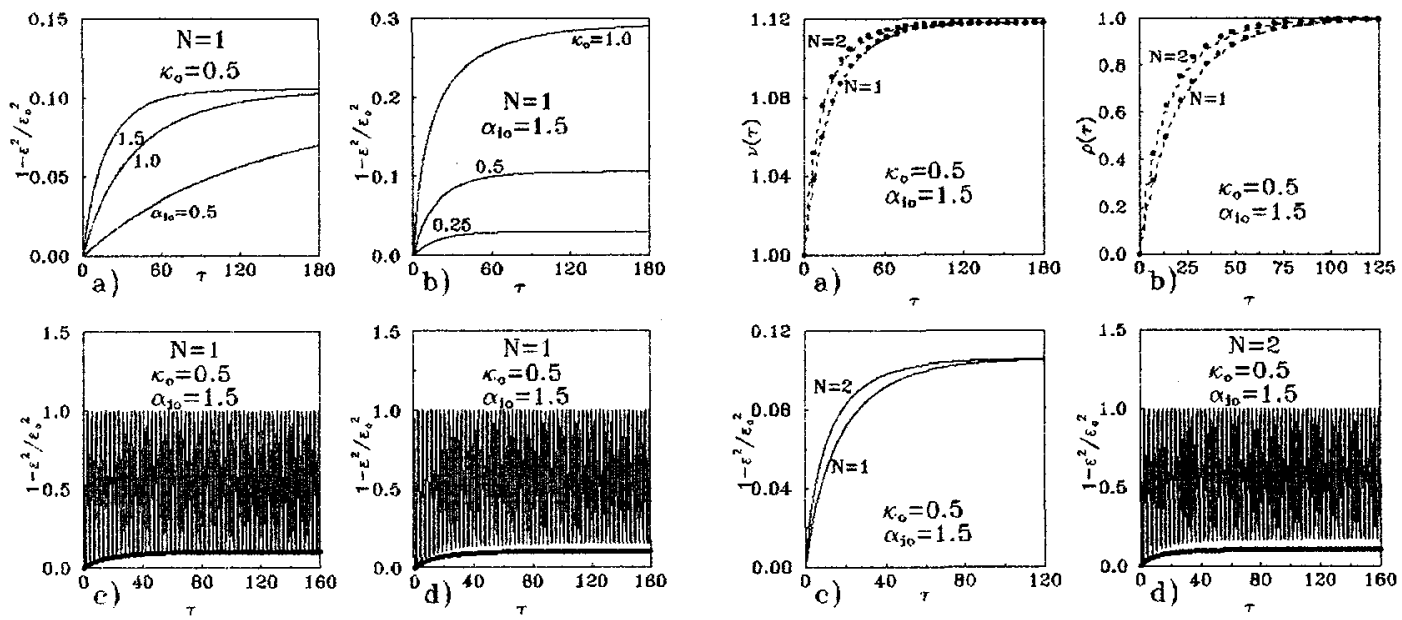

Figure 3: WKB results for the net relative energy loss for one-photon ionization process for: $(\alpha) \alpha_{i o}=0.5,1.0$ and 1.5 and $\kappa_{0}=0.5 ;$ (b) $\kappa_{0}=0.25,0.5,1.0$ and $\alpha_{i 0}=1.5$; (c) WKB results for the net relative energy loss (thick solid curve) and the relative fast electric field energy deviation from its initial value (oscillating thin solid curve) for $\alpha_{i o}=1.5$ and $\kappa_{0}=0.5$; (d) WKB result for the net relative energy loss (thick solid curve) versus the exact numerical one for the relative fast electric field energy deviation from its initial value (oscillating thin solid curve) for the same choice of $\alpha_{i o}$ and $\kappa_{0}$ as in (c).
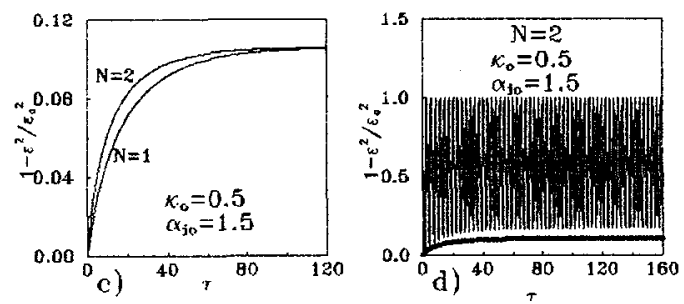

Figure 4: Comparison of various results for one-photon and two-photon ionization processes for $\alpha_{i o}=1.5$ and $\kappa_{0}=0.5$ : (a) Frequency $v$ as a function of time $\tau$; (b) Plasma density $\rho$ as a function of time $\tau$. The solid dots and the dashed curves refer to the WKB and the exact numerical solutions respectively. (c) WKB results for the net relative energy loss; (d) WKB result for the net relative energy loss (thick solid curve) versus the exact numerical one for the relative fast electric field energy deviation from its initial value (oscillating thin solid curve) for the two-photon ionization process.

In Fig. 3b, the net relative energy loss is shown, for three different values of the plasma parameter $\left(\kappa_{0}=0.25,0.5\right.$ and 1.0) and fixed value of $\alpha_{i o}\left(\alpha_{i o}=1.5\right)$; its time asymptotic values are 
$0.03,0.1$ and 0.29 respectively. The WKB result for the net relative energy loss (thick solid curve) and the relative fast electric field energy deviation from its initial value (oscillating thin solid curve) for the particular choice of $\alpha_{i o}=1.5$ and $\kappa_{0}=0.5$ is shown in Fig. 3c. It is seen that this approximate solution has an oscillating "reactive" part, which does not contribute to the net energy loss, and a "resistive" part, due to the dissipation of the WKB (slowly-varying) envelope. On the other hand, in Fig. 3d the WKB result for the net relative energy loss (thick solid curve) is shown, versus the exact numerical one for the relative fast electric field energy deviation from its initial value (oscillating thin solid curve) for the same choice of $\alpha_{i O}$ and $\kappa_{O}$. As it is observed, the WKB result (near $10 \%$ ) is in a relatively good agreement with the numerical one (near $15 \%$ ).

In Fig. 4, various results are compared for one-photon $(N=I)$ and two-photon ionization processes $(N=2)$ for $\alpha_{i o}=1.5$ and $\kappa_{o}=0.5$. Thus, the frequency $v$ and the density $\rho$ are shown as functions of the time $\tau$ in Figs. $4 \mathrm{a}$ and $4 \mathrm{~b}$ respectively. It is readily seen that the two-photon process is faster than the one-photon one, for this particular choice of $\alpha_{i o}>1$. Notice that in both Figs. $4 \mathrm{a}$ and $4 \mathrm{~b}$, the solid dots and the dashed curves refer to the WKB and the exact numerical solutions respectively. The WKB results for the net relative energy loss, which is found to be near $10 \%$ for $\alpha_{i o}=1.5$ and $\kappa_{O}=0.5$, are presented in Fig. 4c. Finally, Fig. $4 \mathrm{~d}$ shows the WKB result for the net relative energy loss (thick solid curve) versus the exact numerical one for the relative fast electric field energy deviation from its initial value (oscillating thin solid curve) for the two-photon ionization process. As in the case of the one-photon process, it is observed that the WKB result, which is near $10 \%$, is in a relatively good agreement with the numerical one, which is near $16 \%$.

In conclusion, the evolution of the wave frequency and its upshift predicted by the WKB approximation are in very good agreement with the exact numerical results. The same applies, though to a lesser degree, for the field energy loss as well.

\section{Acknowledgements}

This work was supported by the Commission of the European Union through the HCM-Network "Microphysics of Nonlinear Phenomena in Collisionless Plasmas. Application to Space and Laboratory Plasmas", Contract No. CHRX-CT-93-0356.

\section{References}

[1] Wilks S. C., Dawson J. M., Mori W. B., Katsouleas T. and Jones M. E., Phys. Rey. Lett. 62 (1989) 2600-2603.

[2] Savage R. L. Jr, Brogle R. P., Mori W. B. and Joshi C., IEEE Trans.Plasma Sci.21 (1993) 5-19.

[3] Wood W. M., Siders C. W. and Downer M. C., IEEE Trans. Plasma Sci., 21 (1993) 20-33.

[4] Mendonca J. T. and Oliveira e Silva L., Phys. Rev. E 49 (1994) 3520-3523.

[5] Mori W. B., Phys. Scripta T52 (1994) 28-35.

[6] Wilks S. C., Dawson J. M. and Mori W. B., Phys. Rev. Lett. 61 (1988) 337-340.

[7] Yablonowitch E., Phys. Rev. Lett. 31 (1973) $877-879$.

[8] Yablonowitch E., Phys. Rev. Lett. 32 (1974) 1101-1104.

[9] Kuo S. P., Phys. Rev. Lett. 65 (1990) 1000-1003.

[10] Joshi C., Clayton C. E., Marsh K., Hopkins D. B., Sessler A. andWhittum D., IEEE Trans. Plasma Sci.18 (1990) 814-818.

[11] Kuo S. P. and Ren A., IEEE Trans. Plasma Sci. 21 (1993) 53-56.

[12] Goteti V. R. and Kalluri D. K., IEEE Trans. Plasma Sci. 17 (1989) 828-833.

[13] Kalluri D. K., IEEE Trans. Plasma Sci. 21 (1993) 77-81.

[14] Banos A. Jr., Mori W. B. and Dawson J. M., IEEE Trans. Plasma Sci. 21 (1993) 57-69.

[15] Gildenburg V. B., Kim A. V., Krupnov V. A., Semenov V. E., Sergeev A. M. and Zharova N. A., IEEE Trans. Plasma Sci. 21 (1993) 34-44.

[16] Kim A. V., Lirin S. F., Sergeev A. M., Vanin E. V. and Stenflo L., Phys. Rev. A 42 (1990) 2493-2495.

[17] Mendonca J. T. and Oliveira e Silva L., IEEE Trans. Plasma Sci., to appear. 